\title{
Pensamiento crítico a través de un caso de enseñanza: una investigación de diseño educativo
}

\section{Critical thinking through a teaching case: An educational design research}

\author{
MARÍA Luisa Morales BAUTiSTA* \\ FRIDA DÍAZ-BARRIGA ARCEO**
}

El objetivo de este artículo es analizar el desempeño de las habilidades de pensamiento crítico: evaluación y toma de postura en torno al tema de la violencia en las relaciones de pareja adolescente a través de un caso de enseñanza en formato electrónico $e$-caso. La investigación se basó en una metodología de diseño educativo con método mixto y participaron 30 estudiantes de primer semestre de una escuela pública de educación media superior: 20 mujeres y 10 hombres de entre 15 y 16 años. Los resultados muestran que el $e$-caso suscitó la posibilidad de que el estudiantado cuestionara sus ideas respecto a una relación de pareja con tintes de violencia e identificara pros y contras. Esto originó una gama de soluciones para el dilema expuesto en la narrativa del caso que abonan a la transformación de los paradigmas receptivos tradicionales y promueven, así, habilidades críticas en estudiantes.

An educational design investigation was carried out with a group of 30 first semester students from a public high school, 20 women and 10 men between 15 y 16 years old participated. The objective was to analyze the performance of critical thinking skills: evaluation and taking position on the issue of violence in adolescent partner relationships through a case study in electronic e-case format. For this purpose, this research was designed under a mixed method educational design methodology. The results show that the e-case raised the possibility that the students questioned their ideas regarding a relationship with violence tinges, identifying pros and cons, managing to generate a range of solutions for the dilemma exposed in the case narrative. Thus, promoting the transformation of traditional receptive paradigms generating critical skills in students.

\section{Palabras clave:}

pensamiento crítico, investigación de diseño educativo, caso de enseñanza, adolescentes

\section{Keywords:}

critical thinking, educational design research, teaching case, adolescents

Recibido: 24 de septiembre de 2020 | Aceptado para su publicación: 20 de mayo de 2021 | Publicado: 28 de mayo de 2021

Recuperado de: https://sinectica.iteso.mx/index.php/SINECTICA/article/view/1215 doi: 10.31391/S2007-7033(2021)0056-016

\footnotetext{
* Maestra en Docencia para la Educación Media Superior en Ciencias Sociales por la Universidad Nacional Autónoma de México. Doctoranda en Psicología por la misma universidad. Integrante del Grupo de Investigación en Docencia, Diseño Educativo y TIC GIDDET. Líneas de investigación: pensamiento crítico, violencia en la pareja y diseño de casos de enseñanza. Correo electrónico: marialuisamoralesbautista@hotmail.com/ https://orcid.org/0000-0002-8513-8070

** Doctora en Pedagogía por la Universidad Nacional Autónoma de México. Profesora de la Facultad de Psicología de la misma universidad. Miembro del Sistema Nacional de Investigadores nivel 3. Coordina el Grupo de Investigación en Docencia, Diseño Educativo y TIC GIDDET. Líneas de investigación: estrategias de enseñanza-aprendizaje, currículo, diseño de dispositivos pedagógicos basados en la narrativa, trayectorias académicas, inclusión educativa, entre otras. Correo electrónico: diazfrida@prodigy.net.mx/ https://orcid.org/0000-0001-8720-1857
} 


\section{INTRODUCCIÓN}

T a investigación contemporánea sobre el pensamiento crítico en el ámbito educativo se centra, primordialmente, en los juicios emitidos en torno a contenidos disciplinares propios del currículo escolar con un fuerte acento en las habilidades cognitivas del sujeto. Este abordaje ha sido fructífero en cuanto a la comprensión de las habilidades de pensamiento implicadas y a su desarrollo mediado por la escolaridad, pero ha dejado de lado la comprensión y promoción del pensamiento crítico acerca de la implicación del sujeto desde la perspectiva de las creencias e ideología que le es propia, o bien, sus repercusiones para la vida cotidiana, la presión social y cuestiones como el género o posición de clase que ejercen influencia al emitir un juicio crítico (Pacheco y Díaz-Barriga, 2006).

En este sentido, se han encontrado comentarios que cuestionan los estudios sobre el pensamiento crítico que se desvinculan de las intenciones y los propósitos humanos, y no priorizan la contextualización (Carretero, 2012). Por ello, no basta con un adiestramiento de habilidades de pensamiento en aislado, como procesos de cognición en frío (cold cognition), que ignoran el papel que tienen el contexto, las creencias y el contenido de los asuntos humanos y sociales que generan controversia como problemas de la vida cotidiana.

Por lo anterior, hemos optado por una investigación de diseño educativo (Plomp, 2007), en la cual se priorice un estudio situado, es decir, considere el contexto y las particularidades de la población elegida para atender el logro de una meta pedagógica, en este caso, analizar algunas habilidades del pensamiento crítico ante una situación problema a través de un caso de enseñanza.

La investigación de diseño educativo ayuda a entender las relaciones entre la teoría y la práctica y, a la par, aporta conocimiento a la o las teorías en que se sustentan. Este tipo de enfoque permite generar diseños flexibles de entornos de aprendizaje, presenciales, virtuales e híbridos, fundamentados en la cognición situada y el constructivismo (Reigeluth y Frick, 2000).

Este enfoque de investigación admite que el estudiantado participe de modo activo en las tareas de diseño educativo de acuerdo con sus intereses y necesidades, al igual que se involucra en el enriquecimiento del o los dispositivos pedagógicos a utilizar. En nuestro estudio optamos por un caso de enseñanza en formato electrónico, ubicada en la enseñanza activa, experiencial y por indagación, que implica actividades, métodos y estrategias que usan los docentes para conseguir una mayor participación (Díaz-Barriga, 2019).

En este artículo exponemos la investigación de diseño educativo con estudiantes de educación media superior con el propósito de analizar algunas habilidades del pensamiento crítico ante una situación problema (la violencia en las relaciones de pareja) por medio de un caso de enseñanza en formato electrónico, considerado un $e$-caso. Así, contribuimos a las directrices de metodologías situadas, críticas y experienciales, pues buscamos que los participantes logren una problematización en contexto del asunto que nos ocupa, con base en una narrativa que refleja situaciones auténticas propias de la vivencia de esta población meta, de ahí que incluyamos una serie de preguntas que permiten evaluar y deliberar al respecto. 


\section{Investigación de diseño educativo}

La investigación de diseño educativo es un estudio sistemático centrado en el diseño, desarrollo y evaluación de intervenciones educativas cuyo objetivo es avanzar en el conocimiento sobre las características de las intervenciones y los procesos de diseñarlas y desarrollarlas (Plomp, 2007). Por su parte, Rinaudo y Donolo (2010) indican que se trata de una investigación de campo que interviene en un contexto de aprendizaje particular para atender, mediante un diseño instructivo, el logro de una meta pedagógica explícitamente definida.

Estos diseños son centrales en los esfuerzos para mejorar el aprendizaje, crear conocimiento útil y avanzar en la construcción de teorías sobre el aprendizaje y la enseñanza en ambientes complejos (Design-Based Research Collective, 2003). De acuerdo con Plomp (2007), la investigación de diseño educativo considera tres fases: una preliminar, que implica definir las metas pedagógicas del estudio de diseño, elaborar un diagnóstico contextual con la población meta y el diseño instructivo que debe conducir a alcanzar la meta pedagógica; una de desarrollo o implementación, que considera la secuencia interactiva de la implementación del diseño con la población meta que facilita refinarlo y obtener conjeturas de este; y una de evaluación. Finalmente, se procede al análisis retrospectivo de los datos recabados.

La investigación de diseño educativo es un género de investigación en el que el desarrollo iterativo de soluciones a problemas educativos complejos es un eje central. Los investigadores de diseño educativo se centran en resolver problemas significativos del mundo real y, al mismo tiempo, buscan descubrir nuevos conocimientos que puedan informar el trabajo de otras personas que enfrentan problemas similares (Mckenney \& Revees, 2013).

\section{Pensamiento crítico}

Resulta cardinal que se impulse el pensamiento crítico en el estudiantado, dadas sus implicaciones en su desarrollo integral; le permite un proceso de deconstrucciónreconstrucción de su toma de postura, lo que genera argumentos y contraargumentos que la apoyen o refuten.

Elder y Paul (2001) consideran que el pensamiento crítico no puede restringirse a una habilidad racional del pensamiento que preside la acción, ya que siempre opera un sistema de creencias (puntos de vista, marcos de referencia, visiones del mundo) que la persona ha construido en su contexto en función de sus circunstancias, y ello delimita la diversidad de puntos de vista y roles que asume. No obstante, la persona puede cambiar esos marcos de referencia y descentrarse de posturas egocéntricas cuando delibera sobre otras perspectivas; esto debe ser tomado en cuenta al pretender abordar el pensamiento crítico.

De acuerdo con diversos autores, el pensamiento crítico requiere una problematización, en la que se ven implicadas habilidades como análisis, evaluación, argumentación, entre otras, que otorgan la posibilidad de tomar posturas y emitir juicios, los cuales deben estar argumentados; asimismo, en este proceso se considera un componente contextual y motivacional (Ennis, 1993; Boisvert, 2004; Paul \& Elder, 2006; Facione, 2007; Ennis, 2011; Valenzuela, Nieto y Muñoz, 2014). 
No debe dejarse de lado la doble función que posee el pensamiento crítico: interna y externa. Se habla de función interna porque consiste en el intento por establecer la reflexión como una categoría legítima de creación de conocimiento y a la par de autocrítica respecto al propio pensamiento. Es externa porque el pensamiento crítico también conduce a una crítica a las condiciones humanas, sociales y políticas contemporáneas, y apoya el posicionamiento de la persona como actor social (Cebotarev, 2003).

Los postulados mencionados condujeron a optar por una perspectiva de análisis del pensamiento crítico en el estudiantado congruente con dichas premisas, ya que reconocemos que este pensamiento implica determinadas habilidades cognitivas de análisis y evaluación, así como la capacidad de argumentar y emitir juicios con base en razones y evidencias. En el abordaje del pensamiento crítico es importante generar reflexión y autocuestionamiento sobre las situaciones problema que sean de interés, originen controversia e impliquen al sujeto para que, además de procesar la información, pueda ejercer la criticidad y tomar postura, además de reconocer los ángulos y controversias existentes.

\section{Caso de enseñanza e-caso}

Si bien puede identificarse el surgimiento de la metodología de casos a finales del siglo XIX, a lo largo de varias décadas se ha ido transformando e incorporando en distintas disciplinas. La enseñanza y el aprendizaje mediante casos ha mostrado su potencial en el desarrollo del pensamiento crítico, el análisis basado en hechos o evidencia, la solución de problemas y la toma de posturas (Naumes \& Naumes, 2015).

En esta estrategia se expone al estudiantado a situaciones problema complejos y reales para que desarrolle propuestas conducentes a su análisis y solución. El caso se muestra en forma de narración o historia que contiene una serie de atributos que evidencian su complejidad y multidimensionalidad (Díaz-Barriga, 2006). A partir de una serie de cuestionamientos y con apoyo de información que documenta más a fondo el caso, el estudiantado puede confrontar su conocimiento y sistema de creencias respecto a los hechos y perspectivas del caso, tomar postura y manifestar la debida empatía o comprensión de los asuntos humanos y sociales involucrados. En función del manejo didáctico dado al caso, se puede profundizar en las cuestiones personales y subjetivas, así como en las sociales, culturales o políticas en que se aborda la situación problema subyacente.

Los casos de enseñanza han incorporado la mediación de las tecnologías de la información y la comunicación (TIC), lo que ha posibilitado la creación de ambientes virtuales enriquecidos con información y enlaces, interactividad e inmediatez, recursos multimedia, entre otros, que aportan un mayor realismo y acercamiento divergente. Los casos de enseñanza que han incorporado las TIC son denominados $e$-casos (Coll y Monereo, 2011; Díaz-Barriga, 2006).

Existen investigaciones con casos que han aportado resultados favorables, pues estos encarnan principios que contribuyen a una valiosa experiencia de aprendizaje y análisis; utilizan varias formas de interacción, incluidas discusiones, trabajo en equipo, presentaciones, diálogo socrático, así como la incorporación de 
técnicas de aprendizaje activo (Popil, 2011; Farashah \& Tajeddin, 2018; Rhodes, Wilson \& Rozell, 2020). En la misma línea, los casos mediados por tecnologías han aportado el uso didáctico de estos en entornos sincrónicos o asincrónicos, y han propiciado ambientes que promueven la interactividad, el uso de recursos digitales y la búsqueda de información y análisis (Brooke, 2006; Watson \& Sutton, 2012).

En relación con el pensamiento crítico abordado desde la metodología de casos, se ha demostrado que estos facilitan y promueven el aprendizaje activo, ayudan a la resolución de problemas, además de que fomentan el pensamiento crítico y brindan la posibilidad de indagar sobre las situaciones y los dilemas planteados; asimismo, estimulan el análisis, al demandar del estudiantado proyecciones de su actuar ante circunstancias como las expuestas y prepararse para la toma de decisiones y posturas (Brooke, 2006; Popil, 2011). Por su parte, Vargas, González y Navarrete (2018) han tenido resultados que aportan evidencia de la eficacia de trabajar con casos en una investigación de diseño educativo para el fomento de pensamiento crítico.

Con esta investigación se abona a la transformación de los paradigmas receptivos tradicionales. No es de extrañar que universidades prestigiosas internacionales hayan diseñado portales educativos con casos de enseñanza en formatos electrónicos (Universidad de California en Santa Bárbara, 2002; Universidad de Harvard, 2019; Universidad de Delaware, 2020).

\section{MÉTODO}

\section{Objetivo}

Analizar el desempeño de las habilidades de pensamiento crítico: evaluación y toma de postura de estudiantes de educación media superior en su contexto escolar en torno al tema de la violencia en las relaciones de pareja adolescente a través de un caso de enseñanza en formato electrónico ( $e$-caso).

\section{Tipo de estudio}

Se trata de una investigación de diseño educativo centrada en el diseño, desarrollo y evaluación de intervenciones educativas (Plomp, 2007). Es una metodología de campo educativo interesada en mejorar prácticas en diferentes niveles, contextos y disciplinas; la intervención se realiza en un contexto de aprendizaje particular, situado y real (Rinaudo y Donolo, 2010). Este análisis investigativo utiliza un método mixto que combina análisis cuantitativos y cualitativos (Creswell \& Plano, 2018). Los métodos mixtos permiten la obtención de una mejor evidencia y comprensión de los fenómenos y, por ello, facilitan el fortalecimiento de los conocimientos teóricos y prácticos (Zulay, 2011). En este sentido, por un lado, se obtienen resultados de la rúbrica diseñada para ese fin, que considera los niveles de desempeño del estudiantado, y se complementa con un análisis cualitativo desde una perspectiva descriptiva interpretativa de las respuestas escritas (Miles, Huberman \& Saldaña, 2014). 


\section{Participantes}

En el estudio participaron 30 estudiantes de un grupo de primer semestre de educación media superior de una escuela pública; de estos, 20 eran mujeres y 10, hombres, cuyas edades iban de los 15 a los 16 años, todos provenientes del sureste de la Ciudad de México.

\section{Instrumentos}

El $e$-caso, diseñado ex profeso para esta investigación y puesto en línea en una página HTML, se titula “¿Duele más tenerte que dejarte ir?”. Centra su trama en los personajes Zoe y Andrik, dos adolescentes de bachillerato inmersos en una relación de pareja con tintes de violencia que enfrentan un dilema. El $e$-caso permite a los lectores evaluar y tomar postura en torno a lo relatado en la situación concreta para entender su complejidad desde distintas perspectivas y buscar posibles soluciones y conductas de prevención o protección. El $e$-caso se encuentra en la siguiente liga https://marialuisamoralesb.wixsite.com/caso

Para la valoración del pensamiento crítico, creamos y validamos un instrumento que evalúa cuatro habilidades: análisis, evaluación, toma de postura y autorreflexión (en este artículo solo reportamos lo concerniente a la evaluación y la toma de postura). El instrumento cuenta con la explicación de cada dimensión y su conceptualización; asimismo, cada dimensión incluye una serie de ítems (18 en total) que fueron valorados en términos de suficiencia, coherencia, relevancia y claridad con una escala tipo Likert que considera cuatro niveles de desempeño. Las preguntas fueron incluidas en las actividades del caso de enseñanza.

Para la evaluación de las respuestas de los estudiantes, creamos y validamos una rúbrica, que considera las mismas habilidades del pensamiento crítico; para la validación, diseñamos un formato que incluye las dimensiones y los indicadores. Esta rúbrica fue valorada en términos de suficiencia, claridad, coherencia, relevancia y discriminación en una escala dicotómica.

Para la validación del instrumento y la rúbrica, trabajamos con diez especialistas en psicología educativa, a quienes les presentamos el caso de enseñanza en el sitio web; les explicamos el objetivo del $e$-caso y les mostramos, en un primer momento, el instrumento y, en otro, la rúbrica para sus respectivas validaciones. Finalmente, realizamos un análisis de validez de contenido por jueceo de expertos con el modelo propuesto por Tristán (2008), que considera como criterio aceptable aquel cuyo CVR sea mayor o igual a 0.58 . Hicimos las respectivas modificaciones para proceder con una segunda validación, de la cual obtuvimos resultados favorables.

\section{Aspectos éticos}

Mediante el acercamiento a la subdirección del plantel, comenzamos el protocolo para el trabajo en campo, es decir, para la implementación del $e$-caso. Revisamos los horarios disponibles de una de las salas de cómputo a la par de los horarios de los grupos de primer semestre. Considerando la viabilidad de tiempos y espacios, elegimos un grupo y dialogamos con los estudiantes y los padres de familia para 
exponerles el propósito de la actividad. Entregamos los formatos para la recolección de firmas tanto del estudiantado como de los padres y madres de familia para obtener el asentimiento y consentimiento informado, respectivamente.

\section{Procedimiento}

El trabajo con el $e$-caso se planteó de acuerdo con las tres fases de una investigación de diseño educativo: fase preliminar, fase de desarrollo e implementación y fase de análisis.

Fase preliminar. La elaboración del caso surge en un primer momento como respuesta a la necesidad explícita de generar pensamiento crítico en los estudiantes (Unesco, 2016), considerando los altos índices de violencia en la pareja (ENDIREH, 2016); pusimos especial atención en el análisis del contexto educativo del estudio. Aplicamos una entrevista semiestructurada con el objeto de profundizar en los ámbitos de interés - pues esta se caracteriza por ser flexible, dinámica y no directiva (Díaz et al., 2013)- a cinco estudiantes (tres mujeres y dos hombres de 15 y 16 años de edad); las habilidades de interés fueron: análisis, evaluación y toma de postura sobre una relación violenta, así como sus conocimientos previos y creencias al respecto.

Encontramos que sus conocimientos previos son retomados, principalmente, por experiencias previas de violencia en ellas y ellos mismos o en jóvenes cercanos, sobre todo en sus amistades. Creen que la violencia es ejercida de manera unidireccional de hombres hacia mujeres; en la misma línea, exponen que es complejo determinar qué hacer ante una situación de esta índole, por lo que consideran la necesidad de actividades diversas que permitan analizar la prevención y la acción en la escuela, pues estos temas son conversados entre sus amistades, pero en ninguna clase se analizan a fondo.

Para la construcción del caso, tomamos en cuenta tanto la información obtenida como las prescripciones de diseño instruccional de casos de enseñanza en formato electrónico revisadas en la literatura. Consultamos como referente los modelos de diseño instruccional de la Universidad de California en Santa Bárbara (2002), de los $e$-casos del Grupo de Investigación en Docencia, Diseño Educativo y TIC (GIDDET, 2018) y las prescripciones de Wasserman (1994).

Fase de implementación. El trabajo de implementación del e-caso se efectuó durante las seis sesiones consideradas, cada una de las cuales tuvo una duración de tres horas, en las que se llevó a la práctica la metodología de casos. Se destacó la lectura crítica de la narrativa y la resolución de las preguntas guía que posibilitaron analizar y evaluar la situación de la relación de pareja planteada. Las sesiones se llevaron a cabo en la sala de cómputo de la escuela.

Fase de análisis. Creamos una base de datos con las respuestas del estudiantado, las cuales fueron valoradas a través de la rúbrica diseñada para ello; también procedimos al análisis cualitativo de las respuestas y, finalmente, a la discusión de los resultados. 


\section{RESULTADOS}

A través del diseño de las preguntas incorporadas en las actividades del caso de enseñanza, indagamos la evaluación de la situación expuesta para manifestar puntos de vista y posturas asumidas ante el caso. En este escrito presentamos los resultados de la habilidad de evaluación y toma de postura. La evaluación implica comparar una situación real con una ideal; en este sentido, las situaciones reales se destacan ante el ojo crítico de quien evalúa y se puede discernir si una alternativa ofrece mayores ventajas o viabilidad (Garza y De la Garza, 2010, p. 60).

Con relación a la evaluación, un aspecto importante es la utilización de fuentes de indagación. Al respecto, Kriscautzky y Ferreiro (2014) indican que esta tiene que ver con características que debe poseer la información o el mensaje para ser confiable o creíble.

La evaluación se centró en la identificación de las características de la situación planteada, la utilización de fuentes, los pros y contras de la situación, y el contraste de la situación planteada con la ideal a fin de identificar similitudes y diferencias. Los resultados obtenidos con 30 estudiantes mediante la valoración con la rúbrica se muestran en la tabla 1.

Tabla 1. Resultados de la habilidad de evaluación del caso de enseñanza

\begin{tabular}{|c|c|c|c|c|c|c|}
\hline Dimensión & Criterio & Sexo & Nivel alto & $\begin{array}{c}\text { Nivel } \\
\text { moderado }\end{array}$ & Nivel bajo & $\begin{array}{l}\text { No cumple con } \\
\text { el criterio }\end{array}$ \\
\hline \multirow{18}{*}{ Evaluación } & \multirow{3}{*}{$\begin{array}{l}\text { Reconocimiento } \\
\text { de elementos de } \\
\text { la presencia de } \\
\text { violencia mutua } \\
\text { en la pareja }\end{array}$} & Total & 3 & 19 & 8 & 0 \\
\hline & & Mujeres & $2(10 \%)$ & 12 (60\%) & $6(30 \%)$ & $0(0 \%)$ \\
\hline & & Hombres & $1(10 \%)$ & 7 (70\%) & $2(20 \%)$ & $0(0 \%)$ \\
\hline & \multirow{3}{*}{$\begin{array}{l}\text { Utilización de } \\
\text { fuentes para } \\
\text { realizar la } \\
\text { evaluación }\end{array}$} & Total & 0 & 4 & 20 & 6 \\
\hline & & Mujeres & $0(0 \%)$ & $4(20)$ & $13(65 \%)$ & $3(15 \%)$ \\
\hline & & Hombres & $0(0 \%)$ & $0(0 \%)$ & 7 (70\%) & $3(30 \%)$ \\
\hline & \multirow{3}{*}{$\begin{array}{l}\text { Identificación } \\
\text { de los pros de la } \\
\text { situación }\end{array}$} & Total & 2 & 12 & 10 & 6 \\
\hline & & Mujeres & $2(20 \%)$ & $9(40 \%)$ & 7 (35\%) & $2(10 \%)$ \\
\hline & & Hombres & $0(0 \%)$ & $4(30 \%)$ & $2(20 \%)$ & $4(40 \%)$ \\
\hline & \multirow{3}{*}{$\begin{array}{l}\text { Identificación de } \\
\text { los contras de la } \\
\text { situación }\end{array}$} & Total & 6 & 15 & 9 & 0 \\
\hline & & Mujeres & $3(15 \%)$ & $13(65 \%)$ & $4(20 \%)$ & $0(0 \%)$ \\
\hline & & Hombres & $3(30 \%)$ & $2(20 \%)$ & $5(50 \%)$ & $0(0 \%)$ \\
\hline & \multirow{3}{*}{$\begin{array}{l}\text { Contrastación } \\
\text { de la situación } \\
\text { planteada con la } \\
\text { situación ideal } \\
\text { identificando } \\
\text { similitudes }\end{array}$} & Total & 2 & 11 & 10 & 7 \\
\hline & & Mujeres & $2(10 \%)$ & $6(30 \%)$ & $9(45 \%)$ & 3 (15\%) \\
\hline & & Hombres & $0(0 \%)$ & $5(50 \%)$ & $1(10 \%)$ & $4(40 \%)$ \\
\hline & \multirow{3}{*}{$\begin{array}{l}\text { Contrastación } \\
\text { de la situación } \\
\text { planteada con la } \\
\text { situación ideal } \\
\text { identificando } \\
\text { diferencia }\end{array}$} & Total & 13 & 12 & 5 & 0 \\
\hline & & Mujeres & $9(45 \%)$ & $9(45 \%)$ & $2(10 \%)$ & $0(0 \%)$ \\
\hline & & Hombres & $4(40 \%)$ & $3(30 \%)$ & $3(30 \%)$ & $0(0 \%)$ \\
\hline
\end{tabular}


En un primer momento, la pregunta fue ¿consideras que en esta relación hay violencia? Los 30 estudiantes indicaron que sí, pero tres hicieron algunas aclaraciones. La $h$ refiere que se trata de un estudiante hombre y la $m$, de una estudiante mujer:

(h) No es como tal violencia física si no que violencia con palabras agresiones y faltas de respeto.

(m) Si, aunque no lo parezca.

(h) Sí hay violencia no a golpes, pero sí hay.

Estas aclaraciones permiten visualizar lo que se ha dicho sobre la violencia psicológica: que no es visible, es "tenue" o "sutil", y se minimiza respecto a la violencia física, que sí es visible.

En cuanto a los argumentos que sustentan el porqué de la existencia de violencia en la pareja de la historia narrada, encontramos que es en el nivel moderado donde se concentran las respuestas de los estudiantes:

(m) Se hallan en ella varios indicadores de una violencia psicológica, como lo son los chantajes, las groserías cuando se ignoran por mencionar algunas, de ambas partes, muchas veces es difícil detectar este tipo de violencia porque no deja ninguna marca visible como lo dice la especialista que leí pero hay que estar atento a lo que nos pase o pase a los amigos.

(h) Pues porque como tal no hay golpes o algo así, todo es más verbal, ambos se hacen mal y se dicen cosas hirientes incluso se prohíben.

(m) La violencia no solo puede ser por medio físico sino también psicológica pues en ella se abusa lo emocional, verbal y el maltrato psicológico. En este caso hay gritos, manipulaciones y celos de Andrik, pero también de Zoe incluso de sus amigos.

El principal elemento reconocido en este nivel de desempeño es la identificación de la violencia mutua en la narrativa del $e$-caso. En este sentido, es menester subrayar que, históricamente, la violencia ha sido sobre las mujeres; son más que sabidas las cifras nacionales e internacionales al respecto (Organización de las Naciones Unidas-México, 2018); no minimizando este hecho, Segato (2003) retoma las palabras de un informe del Programa de las Naciones Unidas para el Desarrollo: "Ninguna sociedad trata a sus mujeres tan bien como a sus hombres" (p. 132). Así, es necesario no olvidar la historia de nuestra sociedad patriarcal, pero también es fundamental visibilizar las interacciones de una relación de pareja con violencia mutua, cuestión que se plasmó en la narrativa y que el estudiantado pudo reconocer.

Respecto a la utilización de las fuentes de referencia, en el nivel más alto de desempeño se espera que la información de cualquier fuente deba revisarse y cuestionarse, lograr arribar a su interpretación, encontrar intereses dobles en los mensajes si fuera el caso, dilucidar sobre los puntos de vista de los expertos de dichas fuentes, etcétera. Sin embargo, el estudiantado se ubicó en el nivel bajo de desempeño en esta habilidad, puesto que la información de las fuentes de consulta no suele cuestionarse ni corroborarse o cruzarse con otra información ni tampoco preguntar sobre su intención; no hay suficiente interpretación de los mensajes para desarrollar el análisis. Los puntos de vista de los expertos se toman, principalmente, como hechos verídicos, sin cuestionamiento alguno. 
La identificación de los pros de la situación se encuentra en el nivel moderado con base en las respuestas de los participantes. Se identifican algunos pros de la situación en cuanto a la apertura al diálogo y la resolución de conflictos:

(m) Al final desean hablar para ver qué está pasando, hay amor de por medio al igual que el dinero y pues se sienten atraídos físicamente el uno al otro.

(m) Que se dicen la verdad, es mejor la verdad que duela que la mentira que oculte y a veces hablan y sobre todo esta última vez para ver qué pasa.

(m) Mi opinión es que en esta relación hay muy pocos pros ya que no es una buena relación, pero uno de los pros sería que si se quieren y quieren pasar más tiempo juntos y van a hablar sobre lo que pasará.

Si bien se identifica la posibilidad del diálogo y la resolución a los conflictos, resaltan las ideas románticas e idealizadas de amor, lo cual podría repercutir en la capacidad para reconocer y denominar la violencia vivida, lo que imposibilita desentrañar ideas arraigadas de ese tipo de amor romántico (Hernández, Castro y Barrios, 2020).

Las respuestas respecto al indicador de identificación de los contras se ubican en el nivel moderado, debido a que los alumnos y alumnas exponen esos contras con argumentos válidos que hacen referencia a la violencia narrada y a sus diversas manifestaciones:

(h) Desconfianza, maltrato, infidelidad, incongruencia y falta de comunicación que los daña a ambos.

(m) El chantaje, uso excesivo de su tiempo, faltas de respeto, ella pierde clases y llora, él se siente enfadado y mal.

(m) Al no saber cómo quererse y al no estar preparados para una relación, se hablan feo, se humillan, se controlan.

Los participantes identifican los principales contras de la relación con argumentos que hacen referencia a la violencia, pero también hablan del "no saber cómo quererse" vinculado nuevamente a las ideas de amor romántico.

En el indicador de contrastación de la situación planteada con la ideal, que identifica similitudes, encontramos que las respuestas se ubican en el nivel moderado. El alumnado reconoce ambas situaciones, la ideal y la real, lo que le permite comparar las dos situaciones y hallar una similitud:

(h) Que los dos dialoguen sobre el tema para que lleguen a un acuerdo, por eso se quedan al final de sus clases y puedan encontrar una solución, yo opino que separarse.

(m) Pues solo veo el amor que se tienen, si a eso se le puede llamar amor, porque qué es el amor, lo que sí es su oportunidad a hablar sobre lo que pasa al final de las clases

(h) No encuentro mucha similitud en los dos tipos de relación, pero alguna de ellas podría ser, que todo ese "cariño o amor" que se tienen lo usaran para mejorar la relación, alguna ayuda psicológica, pero justo hablarlo como bien se dice en el caso.

Por su parte, las respuestas del indicador que aborda el contraste de la situación planteada con la ideal y encuentra diferencias se ubican en el nivel alto; en este se 
determina la situación ideal y la situación trazada en el caso, lo que permite comparar ambas y hallar una amplia gama de diferencias. Los estudiantes escriben lo siguiente:

(h) El comportamiento de cada uno de ellos, los valores no se rigen de buena manera, no hay una buena comunicación, hay malos tratos y degradación, en una relación ideal no debería existir eso, si diferencias y discusiones porque son diferentes y no en todo coincidirán, pero por eso existe el diálogo.

(m) Que en la ideal hay paz (por así decirlo ya que no hay maltratos por ninguno de los dos). Y en la del caso tienen muchas diferencias ya que tenían muchos problemas y había discusiones y violencia psicológica que ya vimos que es más que insultar o decir groserías.

(m) Que en una relación ideal existe confianza, amor propio, comprensión, tolerancia etc. y en la relación que tienen Andrik y Zoe es todo lo contrario.

Vislumbramos la variedad de diferencias entre la relación del caso y una que considera la no violencia como forma de interacción. Esto otorga la posibilidad de tener presente la gama de manifestaciones de violencia psicológica, más allá de la únicamente verbal. Otra de las habilidades es la toma de postura asumida ante la situación expuesta en el $e$-caso; las respuestas se muestran en la tabla 2.

Tabla 2. Resultados de la habilidad de toma de postura argumentada del caso de enseñanza

\begin{tabular}{|c|c|c|c|c|c|c|}
\hline Dimensión & Criterio & Sexo & Nivel alto & $\begin{array}{c}\text { Nivel } \\
\text { moderado }\end{array}$ & Nivel bajo & $\begin{array}{l}\text { No cumple } \\
\text { con el } \\
\text { criterio }\end{array}$ \\
\hline \multirow{12}{*}{$\begin{array}{l}\text { Toma de } \\
\text { postura }\end{array}$} & \multirow{3}{*}{$\begin{array}{l}\text { Generación de una } \\
\text { postura argumentada }\end{array}$} & Total & 1 & 20 & 8 & 1 \\
\hline & & Mujeres & $0(0 \%)$ & $18(90 \%)$ & $2(10 \%)$ & $0(0 \%)$ \\
\hline & & Hombres & $1(10 \%)$ & $2(20 \%)$ & $6(60 \%)$ & $1(10 \%)$ \\
\hline & \multirow{3}{*}{$\begin{array}{c}\text { Utilización de fuentes } \\
\text { para realizar la } \\
\text { evaluación }\end{array}$} & Total & 0 & 13 & 14 & 3 \\
\hline & & Mujeres & $0(0 \%)$ & $9(45 \%)$ & $9(45 \%)$ & $1(5 \%)$ \\
\hline & & Hombres & $0(0 \%)$ & $4(40 \%)$ & $5(50 \%)$ & $2(20 \%)$ \\
\hline & \multirow{3}{*}{$\begin{array}{l}\text { Identificación de } \\
\text { soluciones }\end{array}$} & Total & 2 & 24 & 2 & 2 \\
\hline & & Mujeres & $2(10 \%)$ & $15(75 \%)$ & $1(5 \%)$ & $2(10 \%)$ \\
\hline & & Hombres & $0(0 \%)$ & $9(90 \%)$ & $1(10 \%)$ & $0(0 \%)$ \\
\hline & \multirow{3}{*}{$\begin{array}{l}\text { Generación de conclu- } \\
\text { siones }\end{array}$} & Total & 1 & 21 & 8 & 0 \\
\hline & & Mujeres & $1(5 \%)$ & $12(60 \%)$ & $7(35 \%)$ & $0(0 \%)$ \\
\hline & & Hombres & $0(0 \%)$ & $9(90 \%)$ & $1(10 \%)$ & $0(0 \%)$ \\
\hline
\end{tabular}

En cuanto al indicador de la toma de postura, las respuestas se concentran en el nivel moderado de la rúbrica, en el que existe una posición específica y se explican argumentos que tienen que ver con la temática. Los estudiantes plantearon lo siguiente:

(m) Estoy en contra de cualquier tipo de violencia en el noviazgo, porque una relación sana o adecuada está establecida o se basa en el respeto, confianza y valoración, y si no hay eso en una relación no es seguro que exista un ambiente sano y agradable para los dos. 
(m) Sé que es una relación con violencia psicológica, actualmente llamado "relaciones tóxicas" aunque algunas veces las parejas toman esto a juego y creen que esto no existe y solo es un invento, pero la realidad es que estas relaciones "tóxicas" llegan a hacer bastante daño a los dos y que mejor que estemos enterados y hagamos algo para no producirla.

(m) Es una relación inestable, pues aunque "cumplen", en cierta forma, con los aspectos de una relación o noviazgo, tienen un gran o enorme problema, no son lo que necesitaban o lo que buscan, cada uno tiene experiencias diferentes y distintas que van cambiando la situación de su relación de peor a catastrófico y se ve con toda la violencia que viven y se hacen los dos y yo estoy a favor de relaciones relax respetándome a mí y a mi pareja.

Advertimos una postura y argumentos en contra de la violencia; los participantes abordan las consecuencias dañinas en ambos miembros de la relación, así como el aumento de la violencia dentro de las relaciones. Cabe indicar que la argumentación tiene como propósito fundamental dar cuenta de la opinión que se tiene a partir de un punto de vista mediante explicaciones razonados que sostienen la postura planteada, lo que constituye un punto para fomentar distintas habilidades de pensamiento crítico, como poseer una claridad de planteamientos, identificar puntos de vista ambiguos, evaluar los argumentos, entre otros.

Si bien se muestra una toma de postura, la utilización de las fuentes se ve deficiente, pues, al igual que en la habilidad de evaluación, se ubica en un nivel bajo, en el cual los puntos de vista de los expertos se toman como válidos, sin cuestionamiento, lo que hace imposible la contraargumentación.

Para el indicador de generación de soluciones, solicitamos que se escribiera un final para la historia; en él se podría ubicar la o las posibles soluciones que el alumnado sugiere. Los finales se sitúan en el nivel moderado; en este sentido, el estudiantado propone una solución que indica la comprensión del problema, y que es sensible a los factores contextuales, así como a una de las siguientes dimensiones: ética, lógica o cultural del problema. A continuación, presentamos uno de los finales:

(m) Andrik y Zoé se dieron cuenta que tenían una relación "tóxica" así que decidieron buscar ayuda profesional, ya que querían solucionar sus problemas y dejar de hacer violencia el uno al otro que fue a causa de lo que vivieron en el pasado [...]. Fueron con un profesional y recibieron la ayuda necesaria [...] ellos cambiaron, pero, aunque se tenían el cariño suficiente, no pudieron ya que ambos estaban marcados por sus errores, y decidieron terminar su relación por el bien de ambos [...] pero ese cariño siempre iba a existir, ya que para ambos fue un amor bonito el cual a pesar de los problemas y discusiones tendrá momentos lindos [...]. y así fue el primer amor, su primer amor que les ayudó a saber cómo no es una relación, pues las relaciones violentas no son lo mejor.

En los finales se observa la posibilidad de pedir apoyo, tanto de un o una especialista como de la familia y amigos, y también se elige por terminar la relación; solo dos finales exponen un desenlace trágico como muestra de las consecuencias de una relación de este tipo.

Las conclusiones obtenidas respecto al final escrito y hecho explícito para el caso se ubican en el nivel moderado; se refleja la evaluación informada del alumno o alumna y se argumenta el motivo del final planteado. Como ejemplo, compartimos algunas conclusiones.

(h) Porque en la lectura no hay final y pues ese final que yo puse para mí está bien ya que no haya final y eso permite que nosotros hagamos conciencia de lo que es mejor y ante 
todo el diálogo es lo esencial sin necesidad de agredir, por eso decidí que terminaran y buscaran apoyo de psicólogos.

(m) Porque eso es lo que yo pienso que sería lo mejor para ambos, ya que si siguen con su relación, podrán salir peor, por la comodidad de los dos, para que no les haga daño, ambos son jóvenes y no hay problema terminar cualquier vínculo, falta mucho por conocer, es importante cuidar la salud primero de uno y por qué no, también la del novio.

(h) Porque en consideración mía es la única forma de salir de una relación donde hay violencia psicológica, terminar desde la primera vez que te hacen daño porque si no, eso puede terminar, pero muy mal de otra forma, incluso con la vida.

En los anteriores fragmentos se argumenta el porqué de la elección del final; se apunta hacia una mirada de salud emocional respecto a la violencia en la pareja; asimismo, se delimita un problema de índole personal y se deja de lado el componente social de una violencia estructural. Nótese que, en lo general, las respuestas se ubican en el plano de la subjetividad interpersonal de la pareja; no se discute la violencia como insumo desde la cultura o los modelos sociales vinculados al machismo, la educación en el amor romántico, la asimetría de las relaciones hombre-mujer o las cuestiones de poder o dominación.

\section{DISCUSIón}

En el ámbito de los estudios realizados sobre el pensamiento crítico se ha encontrado que las estrategias que han mostrado mayor efectividad son la oportunidad de diálogo y la exposición de los estudiantes a problemas y ejemplos auténticos y situados (Abrami et al., 2015). Es de mencionar que este tipo de métodos centra la atención en problemáticas situadas, en las cuales las discusiones dirigidas tienen gran impacto; sin duda, una de las técnicas más utilizadas es el cuestionamiento incluido en la estrategia en este escrito: el caso de enseñanza.

Un elemento fundamental del pensamiento crítico es que el sujeto pueda cuestionar y cuestionarse, y considerar en ello la doble función del pensamiento crítico (Cebotarev, 2003). En las líneas del estudiantado pudimos observar que se preguntan qué es el amor, qué es violencia, lo que puede llevar a confrontar sus creencias sobre esta temática, problematizar y deliberar sobre ellas, intereses centrales en un pensador crítico (Paul \& Elder, 2006).

Los resultados en cuanto al uso de las fuentes corroboran lo encontrado en otro estudio con alumnos y alumnas de bachillerato (Gasca y Díaz-Barriga, 2018), puesto que menos de la mitad de estos valoraron el rigor, la fiabilidad y la credibilidad de las fuentes de información y los autores que consultan.

El plano de discusión se centra en explicaciones intrasubjetivas y emocionales; no se ahonda en un ámbito societal; con ello se deja el componente contextual, parte de una perspectiva de pensamiento crítico situada (Facione, 2007; Díaz-Barriga, 2019).

De acuerdo con Garza y De la Garza (2010), la evaluación que implica comparar una situación real con una ideal ayuda a discernir si una alternativa ofrece mayores ventajas o viabilidad; desde esta mirada, los estudiantes pudieron identificar, en mayor medida, las diferencias de la situación del caso y una ideal. Con ello, hicieron posible identificar factores de riesgo del tema abordado. 
En la toma de postura hay un posicionamiento por parte de los estudiantes que retoma argumentos vinculados a la temática; no así, contrargumentos. Clavijo (2010) explica que no hay una sola posibilidad de argumentos, sino una amplia gama que permite ir más allá del pensamiento dogmático. Es menester indicar que se ha observado que pocos son los estudiantes que se ubican en un nivel alto de pensamiento crítico, lo que es similar a lo encontrado por Olivares y Heredia (2012).

De igual manera, se aporta a las investigaciones referidas a la violencia en la pareja, pues el abordaje desde el pensamiento crítico se ha planteado como una directriz en esta esfera del conocimiento; la necesidad de una mirada reflexiva y crítica sobre temáticas como la violencia en la pareja ha sido expuesta por investigaciones que subrayan la naturalización de manifestaciones y comportamientos de la cultura, lo cual avala la permanencia y transmisión de patrones comportamentales a la hora de resolver conflictos en las relaciones de pareja (Casique y Ferreira, 2006; Noa, Creagh y Durán, 2014).

Finalmente, como mencionan Brooke (2006) y Popil (2011), los $e$-casos demandan del estudiantado proyecciones de su actuar ante las situaciones narradas, lo que los prepara para la toma de decisiones y sus posturas. En nuestra investigación, estas últimas se han vislumbrado en los finales descritos, los cuales han posibilitado un papel activo de los estudiantes, al indagar sobre las situaciones y los dilemas planteados. Ello es una aportación a las investigaciones que retoman los $e$-casos para el abordaje y la promoción del pensamiento crítico en contextos reales y situados mediante investigaciones de diseño educativo.

\section{CONCLUSIONES}

En este artículo mostramos los resultados de implementación de un caso de enseñanza en formato electrónico que aborda el pensamiento crítico centrado en el tema de violencia en la pareja adolescente. Parte de una investigación de diseño educativo, la cual se realizó desde una fase preliminar que permitió explorar a la población meta y el contexto, así como otorgar los cimientos para el diseño tanto del $e$-caso como de los instrumentos de evaluación.

El método de casos facilita trabajar habilidades cognitivas; en este sentido, es menester indicar que no existen respuestas "buenas", "correctas" o "únicas", sino procesos reflexivos que comienzan en el aula e impacten en la vida del estudiantado.

Un elemento clave en el uso de casos de enseñanza como recurso pedagógico son las preguntas de análisis, que constituyen el medio fundamental de la profesora o el profesor para mediar el encuentro del alumnado con el material de estudio. Queda evidenciado que un caso de enseñanza debe tener las posibilidades de ser evaluado y, a partir de ello, contar con la posibilidad de generar y asumir una postura.

Una investigación de diseño educativo admite el manejo de una secuencia iterativa de microciclos de diseño y análisis (Van den Akker et al., 2006). En este ciclo hemos advertido que el $e$-caso viabilizó en los estudiantes evaluar la relación planteada de los personajes, cuestionar sus ideas sobre una relación ideal de pareja, identificar pros y contras, y generar una gama de posibilidades para deliberar sobre dinámicas como la de la historia del caso. 
La investigación de diseño educativo con un tipo de estudio mixto se aleja del enfoque analítico experimental de relaciones simples causa-efecto y variables aisladas, porque permite estudios en ambientes sociales complejos, cuyo propósito es comprender y mejorar los procesos de aprendizaje o trabajar con habilidades cognitivas, que se entienden como procesos situados en configuraciones sociohistóricas particulares.

Finalmente, es de subrayar las limitaciones de esta investigación, en términos de generar posibilidades que vayan más allá de perspectivas en el plano personal; por tanto, se prevé que en próximos ciclos se incorporen rutas que conduzcan a análisis que involucren lo societal y contextual; asimismo, se pretende trabajar con la capacidad de cuestionar las fuentes de consulta para facilitar la contraargumentación. Así, la perspectiva iterativa de ciclos en una investigación de diseño educativo permite ir mejorando los diseños de acuerdo con los objetivos, temáticas y población meta.

\section{Agradecimientos}

Este trabajo es parte de la investigación de la primera autora bajo la dirección de la segunda en el programa de doctorado en Psicología de la Facultad de Psicología de la Universidad Nacional Autónoma de México (UNAM), con apoyo financiado por el Consejo Nacional de Ciencia y Tecnología, becaria 629478, con número de beca 479076. Nuestro agradecimiento al apoyo recibido de la DGAPA-UNAM a través del proyecto PAPIIT IN301620.

\section{REFERENCIAS BIBLIOGRÁFICAS}

Abrami, P., Bernard, R., Borokhovski, E., Waddington, D., Wade, A. \& Persson, T. (2015). Strategies for teaching students to think critically: A meta-analysis. Review of Educational Reserch, vol. 85, núm. 2, pp. 275-314. http://dx.doi. org/10.3102/0034654314551063

Boisvert, J. (2004). La formación del pensamiento crítico. Teoría y práctica. México: FCE.

Brooke, S. (2006). Using the case method to teach online classes: Promoting socratic dialogue and critical thinking skills. International Journal of Teaching and Learning in Higher Education, vol. 18, núm. 2, pp.142-149. Recuperado de https://eric.ed.gov/?id=EJ1068074

Carretero, M. (2012). Cognición y educación. En M. Carretero y J. A. Castorina (comps.). Desarrollo cognitivo y educación I. Los inicios del conocimiento (pp. 89-112). Barcelona: Paidós.

Casique, L. y Ferreira, A. (2006). Violencia contra mujeres: reflexiones teóricas. Revista Latino-am Enfermagem, vol. 14, núm. 6, pp. 1-8. Recuperado de http://rlae.eerp.usp.br/

Cebotarev, E. (2003). El enfoque crítico: una revisión de su historia, naturaleza y algunas aplicaciones. Revista Latinoamericana de Ciencias Sociales, Niñez y Juventud, vol. 1, núm. 1, pp. 1-28. Recuperado de http://revistaumanizales.cinde.org.co/rlcsnj/index.php/Revista-Latinoamericana/article/ view/333/199 
Clavijo. R. (2010). Habilidades de pensamiento crítico en el bachillerato: la capacidad argumentativa. Tesis de maestría, Universidad de Cuenca. Repositorio institucional-Universidad de Cuenca.

Coll, C. y Monereo, C. (2011). Psicología de la educación virtual. España: Morata.

Creswell, J. \& Plano, V. (2018). Designing and conducting mixed methods research. EUA: SAGE.

Design-Based Research Collective (2003). Design-Based Research: An emerging paradigm for educational inquiry. Educational Researcher, vol. 32, núm. 1, pp. 5-8. https://doi.org/10.3102/0013189X032001005

Díaz-Barriga, F. (2019). Dispositivos pedagógicos basados en la narrativa. México: SM.

Díaz-Barriga, F. (2006). Enseñanza situada. Vínculo entre la escuela y la vida. México: McGraw-Hill.

Díaz, L., Torruco, U., Martínez, M. y Varela, M. (2013). La entrevista, recurso flexible y dinámico. Investigación en Educación Médica, vol. 2, núm. 7, pp. 162-167. Recuperado de https://www.redalyc.org/articulo.oa?id=349733228009

Elder, L. \& Paul, R. (2001). Thinking with concepts. Journal of Developmental Education, vol. 24, núm. 3, pp. 42-43. Recuperado de http://www.jstor.org/ stable/42775835

ENDIREH (2016). Información sociodemográfica de las relaciones de pareja en México. Recuperado de https://www.gob.mx/conapo/articulos/informacion-sociodemografica-de-las-relaciones-de-pareja-en-mexico

Ennis, R (2011). The nature of critical thinking: An outline of critical thinking dispositions and abilities. Conference on Thinking at MIT, Cambridge, MA, July 1994. Last revised May 2011.

Ennis, R. (1993). Critical thinking assessment. Theory into practice, 32 (3), 179186. DOI: https://doi.org/10.1080/00405849309543594

Facione, P. (2007). Pensamiento crítico ¿Qué es y por qué es importante? Insight Assessment. Recuperado de https://www.insightassessment.com/article/ pensamiento-critico-que-es-y-por-que-es-importante?lang=es

Farashah, M. \& Tajeddin, M. (2018). Effectiveness of teaching methods in business education: A comparison study on the learning outcomes of lectures, case studies and simulations. The International Journal of Management Education, vol. 16, pp. 131-142. https://doi.org/10.1016/j.ijme.2018.01.003

Garza, R. y De la Garza, R. (2010). Pensamiento crítico. México: CENGAJE Learning.

Gasca, A. y Díaz-Barriga, F. (2018). Lectura en internet. Habilidades para la búsqueda y gestión de información en estudiantes de bachillerato. En D. Hernández, D. Cassany y R. López (coords.). Háblame de TIC. Prácticas de lectura y escritura en la era digital, vol. 5 (cap. 2, pp. 53-74). Red Temática Literacidad Digital en la Universidad. Editorial Brujas y Social TIC.

Grupo de Investigación en Docencia, Diseño Educativo y TIC (GIDDET) (2018). Sitio web oficial. Recuperado de https://grupogiddet.wixsite.com/sitio-oficial

Hernández, Y., Castro, A. y Barrios, E. (2020). Creencias del amor romántico en adolescentes: una intervención desde la investigación-acción. Sinéctica. Revista Electrónica de Educación, núm. 55, pp. 1-12. Recuperado de https://sinectica.iteso.mx/index.php/SINECTICA/article/view/1095 
Kriscautzky, M. y Ferreiro, E. (2014). La confiabilidad de la información en internet: criterios declarados y utilizados para jóvenes estudiantes mexicanos. Educ.Pesqui, vol. 40, núm. 4, pp. 913-934. https://doi.org/10.1590/s151797022014121511

Mckenney, S. \& Revees, T. (2013). Educational design research. En J. Spector et al. (eds.). Handbook of Research on Educational Communications and Technology (4a.). pp. 131-140. https://doi.org/10.1007/978-1-4614-3185-5_11

Milles, M., Huberman, A. \& Saldaña, J. (2014). Qualitative data analysis. A methods sourcebook (3a. ed.). EUA: SAGE Publications.

Naumes, W. \& Naumes, M. (2015). The art and craft of case writing. Nueva York: Routledge.

Noa, L., Creagh, Y. y Durán, Y. (2014). La violencia psicológica en las relaciones de pareja: una problemática actual. Revista Información Científica, vol. 88, núm. 6, pp. 1145-1154. Recuperado de http://revinfcientifica.sld.cu/index. $\mathrm{php} / \mathrm{ric} / \mathrm{article} / \mathrm{view} / 1100 / 2507$

Olivares, S. y Heredia, Y. (2012). Desarrollo del pensamiento crítico en ambientes de aprendizaje basado en problemas en estudiantes de educación superior. Revista Mexicana de Investigación Educativa, vol. 17, núm. 54, pp. 759778. Recuperado de https://www.comie.org.mx/revista/v2018/rmie/index. $\mathrm{php} / \mathrm{nrmie} /$ article/view/327/327

Organización de las Naciones Unidas-México (2018). La violencia contra las mujeres no es normal ni tolerable. Comunicado oficial. Recuperado de https://www. onu.org.mx/la-violencia-contra-las-mujeres-no-es-normal-ni-tolerable/

Pacheco, D. y Díaz-Barriga, F. (2006). Critical thinking in a higher education service-learning program. En K. McKnight Casey, G. Davidson, S. Billig \& N. Springer (eds.). Advancing knowledge in service-learning: Research to transform the ield (pp. 89-110). Greenwich, CT: Information Age Publishing.

Paul, R. \& Elder, L. (2006). The miniature guide to Critical Thinking. Concepts and tools. The Fundation of Critical Thinking. Recuperado de https://www. criticalthinking.org/

Plomp, T. (2007). Educational design research: An introduction. En T. Plomp y N. Nieveen. An introduction to educational design research (pp. 9-35). China: SLO Netherlands Institute for Curriculum Development.

Popil, I. (2011). Promotion of critical thinking by using case studies as teaching method. Nurse Educ Today, vol. 31, núm. 2, pp. 204-207. https://doi. org/10.1016/j.nedt.2010.06.002

Reigeluth, Ch. y Frick, T. (2000). Investigación formativa. Una metodología para crear y mejorar teorías de diseño. En Ch. Reigeluth (ed.). Diseño de la instrucción. Teorías y modelos (parte 2, pp. 181-200). Aula XXI Santillana.

Rinaudo, M. y Donolo, D. (2010). Estudios de diseño. Una perspectiva prometedora en la investigación educativa. Revista Educativa a Distancia (RED), vol. 22, pp. 2-29. Recuperado de https://revistas.um.es/red/article/view/111631

Rhodes, A., Wilson, A. \& Rozell, T. (2020). Value of case-based learning within STEM courses: Is it the method or is it the student? CBE- Life Sciences Education, vol. 19, núm. 3, pp. 19-44. https://doi.org/10.1187/cbe.19-10-0200

Segato, R. (2003). Las estructuras elementales de la violencia. Ensayos sobre género entre la antropología, el psicoanálisis y los derechos humanos. Universidad Nacional de Quilmes. 
Tristán, A. (2008). Modificación al modelo de Lawshe para el dictamen cuantitativo de la validez de contenido de un instrumento objetivo. Avances en Medición, vol. 6, núm. 1, pp.37-48.

Unesco (2016). Habilidades para un mundo cambiante. Aprendizaje y docencia en la Agenda de Educación 2030 (reporte en línea). Recuperado de http:// www.unesco.org/fileadmin/MULTIMEDIA/FIELD/Santiago/pdf/01-EstherCare-ESP-AprendizajeyDocencia.pdf

Universidad de California en Santa Bárbara (2002). The case method of teaching. Teaching with case. Recuperado de http://www.soc.ucsb.edu/projects/casemethod

Universidad de Delaware (2020). Problem based learning clearinghouse. Recuperado de https://www.itue.udel.edu/pbl/problems

Universidad de Harvard (2019). Case program. Recuperado de https://case. hks.harvard.edu/case-teaching-resources/

Valenzuela, J., Nieto, A. y Muñoz, C. (2014). Motivación y disposiciones: enfoques alternativos para explicar el desempeño de habilidades de pensamiento crítico. Revista Electrónica de Investigación Educativa, vol. 16, núm. 3, pp. 1632. Recuperado de https://redie.uabc.mx/redie/article/view/519/956

Van den Akker, J., Gravemeijer, K., McKenney, S. \& Nieven, N. (eds.) (2006). Educational design research. Routledge.

Vargas, I., González, X. y Navarrete, T. (2018). Metodología activa en el estudio de caso para el desarrollo de pensamiento crítico y sentido ético. Enfermería Universitaria, vol. 15, núm. 3, pp. 244-254. https://doi.org/10.22201/ eneo.23958421e.2018.3.65988

Wasserman, S. (1994). El estudio de caso como método de enseñanza. Buenos Aires: Amorrortu Editores.

Watson, S. \& Sutton, J. (2012). An examination of the effectiveness of case method teaching online: Does the technology matter? The International Journal of Management Education, vol. 36, núm. 6, pp. 802-821. https://doi. org/10.1177/1052562912445281

Zulay, P. (2011). Los diseños de MÉTODO MIXTO en la investigación en educación: una experiencia concreta. Revista Electrónica Educare, vol. 15, núm. 1, pp. 15-29. https://doi.org/10.15359/ree.15-1.2 